\title{
Features of Aging of Road Bitumen
}

Myroslava Donchenko ${ }^{1}$, Oleg Grynyshyn ${ }^{2}$, Yuriy Khlibyshyn ${ }^{3}$

1. Department of Chemical Technology of Oil and Gas Processing, Lviv Polytechnic National University,

UKRAINE, Lviv, 12 Bandera street, E-mail: myroslava.i.donchenko@lpnu.ua

2. Department of Chemical Technology of Oil and Gas Processing, Lviv Polytechnic National University,

UKRAINE, Lviv, 12 Bandera street, E-mail: oleg.b.grynyshyn @1pnu.ua

3. Department of Organic Products Technology, Lviv Polytechnic National University, UKRAINE, Lviv,

12 Bandera street, E-mail: yurii.y.khlibyshyn @lpnu.ua

The aging process of oxidized and residual oil bitumens was carried out in a thin film at the temperature of $163^{\circ} \mathrm{C}$ for 5 and 10 hours. By comparing the main characteristics of aged and original bitumen samples it was possible to investigate the changes that occur during aging.

Keywords - bitumen, bitumen aging, tar oxidation, oxidized bitumen, residual bitumen, pavement.

\section{Introduction}

Today, the aging of the binder is one of the main reasons for the loss of bitumen properties. In accordance to thus, the deterioration of the main bonding component of the asphalt mixture leads to deformation and destruction of the entire pavement. The reason for such negative changes is the effect of atmospheric oxygen, solar radiation, water, mechanical loads and high technological temperatures on the thin bituminous film.

Until recently it was believed that the effect of light and ultraviolet radiation can be ignored, but nowadays this claim was found to be false. Water also has a significant negative impact on the service life of the pavement. In the regions with increased humidity, when the roads are sometimes flooded, the processes that accelerate the loss of the pavement strength will certainly occur. In addition, water impairs the distribution of road load, causing surface breaking and loss of fine-grained fractions. Moisture leaches asphalt concrete, dissolves and washes away its components, which contributes to the intensity of bitumen oxidation.

Thus, bitumen constantly undergoes aging due to weather and climatic factors on the one hand, but also undergoes changes due to the processes within its structure. It is important to note that oxygen absorption, which is the reason of bitumen aging, depends on the diffusion rate, bitumen viscosity and the film thickness [1].

So, in this paper we studied the processes occurred on the stationary film during binder aging due to air and high temperature. The samples of residual and oxidized bitumen produced in Ukraine were compared.

\section{Experimental}

To simulate the thermal oxidative aging of bitumen samples, artificial aging was performed according to the method that is slightly modified compared to the standard one and allows to obtain a binder after heating at $163^{\circ} \mathrm{C}$ in a $2 \mathrm{~mm}$ thin film for 5 and $10 \mathrm{~h}$ [18]. As can be seen, the film thickness was changed (cf. $4 \mathrm{~mm}$ according to the standard). However, in this case, the surface of the test sample will be oxidized primarily, whereas the changes in volume will depend on the viscosity [2].

To study we used oxidized bitumen of BND 60/90 brand produced by PJSC "Ukrtatnafta" (Kremenchuk, Ukraine) and residual bitumen of BD 130/200 brand, obtained after refining of Orkhovitska oil at JSC "Ukrgazvydobuvannya" (Lviv, Ukraine).

The weight loss of the samples after aging and the softening temperature changes were determined according to EN 1427:2007. The main indices before and after 5 and $10 \mathrm{~h}$ of aging were determined according to the standard methods, including penetration (EN 1426:2000) and 
ductility (EN 13589:2008). The brittleness temperature $\left(\mathrm{Tb},{ }^{\circ} \mathrm{C}\right)$ was determined by the formula: $\mathrm{Tb}=\mathrm{Ts}-7 \cdot(10-\mathrm{PI})$, where $\mathrm{Ts}$ is the softening temperature, ${ }^{\circ} \mathrm{C}$ and $\mathrm{PI}$ is the penetration index. The plasticity interval of the samples was calculated as the sum of the softening temperature and the brittleness temperature [3]. Group analysis was performed by the Marcusson method.

\section{Results}

The data show a rapid deterioration of the bitumen properties due to aging process. We observed a sharp decrease in the penetration value (by $55.5 \%$ for the oxidized sample and by 26 $\%$ for the residual one), as well as an increase in the softening temperature by $22.5 \%$ and $24.5 \%$ for the oxidized and residual bitumen, respectively. The plastic properties of the oxidized bitumen, the ductility of which is reduced by more than $90 \%$, deteriorate sharply. On the other hand, the plasticity of the residual sample remains at a very high level even after $10 \mathrm{~h}$ of aging. The plastic range of both samples also varies in different ways, namely the temperature range, which is desirable for pavement maintenance and within which the binder remains in the viscoplastic state. In this case, there is a decrease of the value by $9^{\circ} \mathrm{C}$ for the oxidized sample and an increase by $8^{\circ} \mathrm{C}$ for the residual bitumen. Another negative consequence of aging for the oxidized bitumen is the loss of frost resistance (the brittleness temperature increases by almost $20^{\circ} \mathrm{C}$ ). The penetration index is higher for residual bitumen, i.e. this bitumen has better heat resistance [4]. The described characteristics demonstrate that the aging processes that occur in the residual bitumen are less intense to compare with the oxidized sample; however, both bitumen samples after a few hours of aging lose their commercial characteristics.

The data of structural group analysis also confirm the significant impact of aging processes on both samples of bitumen.

\section{Conclusion}

Comparing the aging peculiarities of residual and oxidized bitumen, it can be argued that changes in oxidized bitumen occur with greater intensity. This is especially noticeable due to the analysis of its structure. And, as the result of aging, the structure of the oxidized bitumen becomes similar to the structure of the residual one.

Thus, the aging process leads to a significant deterioration of the binding properties of both samples, but the residual bitumen is more stable. At the same time, even a slight decrease in operational temperature during oxidized bitumen production or certain changes in production technology can significantly improve bitumen properties.

\section{References}

[1] Donchenko, M. I., Grynyshyn, O. B., Kochubey, V. V., Khlibyshyn, Yu. Ya. (2020). Characteristics Of Aging Of Oxidized Oil Bitumen BND 60/90 PJSC "Ukrtatnafta". Chemistry, Technology and Application of Substances, Vol. 3, No. 1, 83-89. https://doi.org/10.23939/ctas2020.01.083.

[2] Pechenyi, B. G., Kurbatov, V. L., Losev, V. P. (2019). On The Mechanism Of Bitumen Aging And Methods Of Their Testing. Universitetskaja nauka, № 2 (8), 28-32.

[3] Kopylov, V. E., Burenina, O. N. (2019). Influence Of Bitumen Plasticity Interval On Asphalt Concrete Thermal Stability. Roads and Bridges, 190-196.

[4] Peide, C., Mingjing, F., Zongwu, Ch., Mingwei, Y. (2018). Residual Fatigue Properties of Asphalt Pavement after Long-Term Field Service. Materials, 11(6), 892-904. https://doi.org/10.3390/ma11060892 